\title{
The Type Specimens of Apoidea (Hymenoptera) Deposited in the Entomological Collection of Instituto Oswaldo Cruz, Rio de Janeiro, RJ, Brazil
}

\author{
Marisa Vianna Ferraz \\ Coleção Entomológica, Departamento de Entomologia, Instituto Oswaldo Cruz, Av. Brasil 4365, 21045-900 \\ Rio de Janeiro, RJ, Brasil
}

A list of 41 Apoidea (Hymenoptera) type specimens deposited in the Entomological Collection of the Instituto Oswaldo Cruz, Rio de Janeiro, RJ, Brazil is presented. The types previously belonged to the Zikán and Schrottky private collections. A total of 11 holotypes and 30 paratypes are listed with their respective data and literature.

Key words: type specimens - Apoidea - Hymenoptera - Zikán Collection - Schrottky Collection Instituto Oswaldo Cruz

The general entomological collection of the Instituto Oswaldo Cruz (IOC), Rio de Janeiro, RJ, Brazil, has approximately 1.2 million specimens, a rich source for research and historical documentation for Brazilian and foreigner researchers. It began in 1900 when researchers on scientific expeditions collected insects vector of human and animal diseases. The collection was under the care of A da Costa Lima until 1964, when he died. Afterwards, Orlando V Ferreira and Sebastião José de Oliveira assumed responsibility for the collection.

The Hymenoptera collection, with approximately 25,000 specimens, has representative species of most families, as well as many nest samples. Two superfamilies of this order, Apoidea and Vespoidea, have species represented by type specimens. This paper deals with the super-family Apoidea.

Some privately owned collections have been integrated into the collection of IOC. The former Zikán Collection includes specimens collected mostly in the National Park of Itatiaia, State of Rio de Janeiro, while the former Schrottky Collection contains specimens collected mostly in Paraguay.

This paper is part of a series of lists being published to cover all orders of insects deposited in the entomological collection of IOC. Lists of type specimens of triatomine bugs (Gonçalves et al. 1994) and mosquitoes (Marchon-Silva et al. 1996) started this series.

Supported by FIOCRUZ/FAPERJ.

Received 7 October 1996

Accepted 19 February 1997
The genera and species are listed alphabetically within an alphabetical arrangement of families and subfamilies. The names were last reviewed and updated by Michener (1954), Stephen (1954), Moure (1960), Ordway (1966), Urban (1967, 1968), Eickwort (1969), Moure and Hurd (1987), and Michener et al. (1994). The information for each species name is provide in the following order: original biomen, author, year of publication, genus, junior synonyms (if any) and type data, including sex, registration number, type locality, collector, date and other informations.

\section{SUBFAMILY PANURGINAE}

Genus Anthrenoides Ducke, 1907

- meridionalis (Schrottky, 1906). Anthrenoides. Synonym of Protandrena meridionalis Schrottky, 1906. Holotype (no. 10), Paraguay: Villa Encarnación; C Schrottky coll. (no coll. data).

Genus Parapsaenythia Friese, 1908

- paspali (Schrottky, 1909). Parapsaenythia. Synonym of Anthrenoides paspali Schrottky, 1909. Holotypes: (no. 07), Paraguay: Puerto Bertoni; C Schrottky coll., 27-XII-1908; (no. 08), Paraguay: Puerto Bertoni, Alto Parana. (no coll. data).

\section{Genus Psaenythia Gerstäcker, 1868}

- atriventris Schrottky, 1907. Psaenythia. Holotype (no. 06), Paraguay: Villa Encarnación; C Schrottky coll., 29-XII-1905.

- burmeisteri Gerstäcker, 1868. Psaenythia. Holotype (no. 04). (no coll. data)

- collaris Schrottky, 1906. Psaenythia. Holotype (no. 14), Paraguay: Villa Encarnación; C Schrottky coll., IX-1904.

- comma Schrottky, 1907. Psaenythia. Holotype 
(no. 05), Paraguay: Villa Encarnación; C Schrottky coll., 12-X-1906.

- physalidis Schrottky, 1907. Psaenythia. Holotype

(no. 13), Paraguay: Villa Encarnación; C Schrottky coll., 13-X-1906.

- picta Gerstäcker, 1868. Psaenythia. Holotype (no. 11), Paraguay: Villa Encarnación; C Schrottky coll., 16-XI-1904.

- quinquefasciata Schrottky, 1907. Psaenythia. Holotype (no. 12), Paraguay: Villa Encarnación; C Schrottky coll., 03-II-1905.

- solani Schrottky, 1907. Psaenythia. Holotype (no. 09), Paraguay: Villa Encarnación; C Schrottky coll., 14-X-1906.

\section{FAMILY ANTHOPHORIDAE SUBFAMILY ANTHOPHORINAE \\ Genus Melissoptila Holmberg, 1884}

- thoracica (Smith, 1854). Melissoptila (Ptilomelissa). Synonym of Melissoptila ochromelaena Moure, 1943. Paratype (no. 24), Brazil: Rio de Janeiro, Itatiaia, 700 m; JF Zikán coll., 29-XI-1935.

Genus Thygater Holmberg, 1903

- chaetaspis Moure, 1941. Thygater (Thygater). Paratype (no. 22), Brazil: Rio de Janeiro, Itatiaia, 720 m, km 12; JF Zikán coll., 05-II-1931.

- sordidipennis Moure, 1941. Thygater (Nectarodiaeta). Paratype (no. 21), Brazil: Rio de Janeiro, Itatiaia, 700 m; JF Zikán coll., 18-VII1931 (in Solanaceae flowers).

\section{SUBFAMILY XYLOCOPINAE}

Genus Ceratinula Moure, 1941

- turgida Moure, 1941. Ceratinula. Paratypes: (no. 25), Brazil: Rio de Janeiro, Itatiaia, $700 \mathrm{~m}$; JF Zikán coll., 07-V-1929 (in Camboata [Myrtaceae] flowers); (no. 26), Brazil: Rio de Janeiro, Itatiaia, 700 m; JF Zikán coll., 20-V-1932 (in Camboata [Myrtaceae] flowers).

- xanthocera Moure, 1941. Ceratinula. Paratype (no. 27), Brazil: Minas Gerais, Mar de Hespanha; JF Zikán coll., 02-IX-1909.

\section{FAMILY COLLETIDAE \\ SUBFAMILY COLLETINAE}

Genus Colletes Latreille, 1805

- albicinctus (Moure, 1943). Colletes. Synonym of Rhynchocolletes albicinctus Moure, 1943. Paratype (no. 28), Brazil: Rio de Janeiro, Itatiaia, 700 m; JF Zikán coll., 26-V-1934 (in Camboata [Myrtaceae] flowers).

\section{SUBFAMILY DIPHAGLOSSINAE}

Genus Ptiloglossa Smith, 1853
- decora Moure, 1945. Ptiloglossa. Paratype (no. 46), Brazil: Rio de Janeiro, Mendes; (no coll. data) XII-1944.

- dubia Moure, 1945. Ptiloglossa. Paratype (no. 47), Brazil: Minas Gerais, Mar de Hespanha; JF Zikán coll., 02-IV-1911.

- immixta Moure, 1945. Ptiloglossa. Paratype (no. 49), Brazil: Rio Grande do Sul, Esteio; R.P.C. Negrini coll., III-1943.

- latecalcarata Moure, 1945. Ptiloglossa. Paratype (no. 44), Brazil: south of Minas Gerais, Virginia, 900 m; JF Zikán coll., 23-III-1919.

- stafuzzai Moure, 1945. Ptiloglossa. Paratype (no. 48), Brazil: São Paulo, Batatais; A.M. Stafuzza coll., III-1943.

- styphlaspis Moure, 1945. Ptiloglossa. Paratype (no. 43), Brazil: São Paulo, Batatais; A.M. Stafuzza coll., XII-1943.

-xanthorhina Moure, 1945. Ptiloglossa. Paratype (no. 50), Brazil: Amazonas, Rio Negro, São Gabriel; JF Zikán coll., 25-X-1927.

\section{SUBFAMILY HALICTINAE}

\section{FAMILY HALICTIDAE}

Genus Augochlora Smith, 1853

- esox (Vachal, 1911). Augochlora (Augochlora). Synonym of Oxystoglossa franzisca Schrottky, 1909; Halictus esox Vachal, 1911; Oxystoglossa schrottkyi Moure, 1940. Paratype (no. 42), Brazil: Rio de Janeiro, Itatiaia, 816 m; JF Zikán coll., 17-VII-1941 (in Azalea flowers).

- pyrgo (Schrottky, 1911). Augochlora (Augochlora). Synonym of Oxystoglossa pyrgo Schrottky, 1911; Halictus liotribes Vachal, 1911. Paratype (no. 41), Brazil: Rio de Janeiro, Itatiaia, 700 m; JF Zikán coll., 20-V-1932 (in Camboata [Myrtaceae] flowers).

- tantilla Moure, 1943. Augochlora (Augochlora). Paratype (no. 19), Brazil: Rio de Janeiro, Itatiaia, 816 m; JF Zikán coll., 15-VII-1941 (in Azalea flowers).

- thusnelda (Schrottky, 1909). Augochlora (Augochlora). Synonym of Oxystoglossa thusnelda Schrottky, 1909. Paratype (no. 20), Brazil: Rio de Janeiro, Itatiaia, 700 m; JF Zikán coll., 28-V1941 (in Poinsettia flowers).

Genus Augochlorella Sandhouse, 1937

- ephyra (Schrottky, 1911). Augochlorella. Synonym of Oxystoglossa ephyra Schrottky, 1911; Halictus ictis Vachal, 1911; Halictus traumatias Vachal, 1911; Oxystoglossa ephyra Moure, 1940; Oxystoglossidia uraniella Moure, 1943. Paratypes: (no. 39), Brazil: Rio de Janeiro, Itatiaia, $700 \mathrm{~m}$; JF Zikán coll., 15-V-1940 (in Poinsettia flowers); (no. 40), Brazil: Rio de Janeiro, Itatiaia, 816 
m; JF Zikán coll., 06-VI-1941 (in Poinsettia flowers).

Genus Augochloropsis Cockerell, 1897

- brachycephala Moure, 1943. Augochloropsis. Paratype (no. 34), Brazil: Rio de Janeiro, Itatiaia, 700 m; JF Zikán coll., 07-XI-1942 (in Ingá flowers).

- nasuta Moure, 1944. Augochloropsis. Paratype (no. 37), Brazil: Rio de Janeiro, Itatiaia, $816 \mathrm{~m}$; JF Zikán coll., 24-VI-1941 (in Azalea flowers).

Genus Habralictus Moure, 1941

- canaliculatus Moure, 1941. Habralictus. Paratype (no. 17), Brazil: Rio de Janeiro, Itatiaia, $816 \mathrm{~m}$; JF Zikán coll., 13-V-1941 (in Poinsettia flowers). - flavopictus Moure, 1941. Habralictus. Paratype (no. 18), Brazil: São Paulo, Rio Claro; Claretiano coll., V-1939; P. F. S. Pereira \& J. A. Mota coll. - orites Moure, 1941. Habralictus. Paratype (no. 38), Brazil: Rio de Janeiro, Itatiaia, 700 m; JF Zikán coll., 12-V-1932 (in Cactus flowers).

Genus Paroxystoglossa Moure, 1940

- transversa Moure, 1943. Paroxystoglossa. Paratypes: (no. 02), probably Paraguay: Puerto Bertoni; coll. ?, 1943 (acquired from the Schrottky collection); (no. 01), probably Paraguay: Puerto Bertoni; (no coll. data) 1947 (acquired from the Schrottky collection).

Genus Pereirapis Moure, 1943

- rhizophila Moure, 1943. Pereirapis. Paratypes: (no. 15), Brazil: Rio de Janeiro, Itatiaia, $700 \mathrm{~m}$; JF Zikán coll., 23-II-1942; ～(no. 16), Brazil: Rio de Janeiro, Itatiaia, $700 \mathrm{~m}$; (no coll. data) 12-V1932 (in Cactus flowers).

Genus Rhinocorynura Schrottky, 1909

- crotonis (Ducke, 1906). Rhinocorynura. Synonym of Halictus (Corynuropsis) crotonis Ducke, 1906; Corynuroides zikani Moure, 1943. Paratype (no. 03), Brazil: Rio de Janeiro, Itatiaia, $700 \mathrm{~m}$; JF Zikán coll., 13-II-1942.

\section{ACKNOWLEDGEMENTS}

To the curator of the Entomological Collection, Instituto Oswaldo Cruz, Dr Sebastião José de Oliveira, for his assistance and supervision. To Ronaldo da Silva Ribeiro for his help and support. To Beatriz Woiski Teixeira Coelho for reviewing the families and genera, and Magaly Dolsan de Almeida for providing incentive and suggestions. To Denise Tavares Gonçalves for English review.

\section{REFERENCES}

Ducke A 1906. Beitrag zur Kenntnis der Solitärbienen Brasiliens. (Hym.). Z Syst Hymenop Dipterol 6: 394400.
Eickwort GC 1969. A comparative morphological study and generic revision of the Augochlorine bees (Hymenoptera: Halictidae). Univ Kansas Sci Bull 48: 325-524.

Gerstäcker A 1868. Psaenythia, eine neue Bienengattung mit gezähnten Schienensporen. Archiv für Naturg a.34: 111-137.

Gonçalves TCM, Almeida MD, Lent H, Jurberg J 1994. Levantamento dos exemplares tipos de Triatomíneos depositados na Coleção Entomológica do Instituto Oswaldo Cruz. Mem Inst Oswaldo Cruz 88: 327333.

Marchon-Silva V, Lourenço-de-Oliveira R, Almeida MD, Silva-Vasconcelos A, Costa J 1996. The type specimens of mosquitoes (Diptera, Culicidae) deposited in the Entomological Collection of the Instituto Oswaldo Cruz, Rio de Janeiro, Brazil. Mem Inst Oswaldo Cruz 91: 471-478.

Michener CD 1954. Bees of Panama. Bull Amer Mus Nat Hist 104: 1-176.

Michener CD, McGinley RJ, Danforth BN 1994. The Bee Genera of North and Central America (Hymenoptera: Apoidea). Smithsonian Institution Press, Washington, $209 \mathrm{pp}$.

Moure JS 1941. Apoidea neotropica - III. Arq Mus Paranaense, Curitiba, 1: 41-99.

Moure JS 1943. Notas sôbre abelhas da Coleção Zikán (Hym. Apoidea). Rev Ent, Rio de Janeiro, 14: 447484.

Moure JS 1944a. Apoidea da coleção do Conde Amadeu A. Barbiellini (Hym. Apoidea). Rev Ent, Rio de Janeiro, 15: 1-18.

Moure JS 1944b. Notas sobre abelhas da Coleção Zikán (Hym. Apoidea). Rev Ent, Rio de Janeiro, 15: 273291.

Moure JS 1945. Contribuição para o conhecimento dos Diphaglossinae, particularmente Ptiloglossa (Hym. Apoidea). Arq Mus Paranaense, Curitiba, 4: 137178.

Moure JS 1960. A review of the genus Paroxystoglossa (Hymenoptera: Halictidae). Univ Kansas Sci Bull 40: 121-133.

Moure JS, Hurd PD 1987. An annotated catalog of the halictid bees of the Western Hemisphere (Hymenoptera: Halictidae). Smithsonian Institution Press, Washington, $405 \mathrm{pp}$.

Ordway E 1966. Systematics of the genus Augochlora north of Mexico. Univ Kansas Sci Bull 46: 509-624.

Schrottky C 1906. Neue und wenig bekannte südamerikanische Bienen. Z Syst Hymenop Dipterol 6: 305-316.

Schrottky C 1907a. Neue und wenig bekannte südamerikanische Bienen. Z Syst Hymenop Dipterol 7: $469-480$.

Schrottky C 1907b. Al conocimiento de los himenópteros del Paraguay. III. An Cient Paraguayos 7(series I): 1-78.

Schrottky C 1909. Nuevos himenópteros sudamericanos. Rev Mus La Plata 16: 137-149

Schrottky C 1911. Descripção de abelhas novas do Brazil e de regiões visinhas. Rev Mus Paulista 8: 7188. 
356 Apoidea Types of Instituto Oswaldo Cruz, RJ, Brazil • MV Ferraz

Smith F 1854. Catalogue of Hymenopterous insects in the collection of the British Museum: 199-465.

Stephen WP 1954. A revision of the bee genus Colletes in America north of Mexico (Hymenoptera Colletidae). Univ Kansas Sci Bull 36: 149-527.

Urban D 1967. As espécies do gênero Thygater Holmberg,
1884. Bol Univ Fed Paraná (Zool) 2: 177-307.

Urban D 1968. As espécies do gênero Melissoptila Holmberg, 1884 (Hymenoptera - Apoidea). Revta bras Ent 13: 1-94.

Vachal J 1911. Etude sur les Halictus d'Amérique. Mis ent Narbonne 19: 53-84. 